\title{
Multiphoton-absorption induced ultraviolet luminescence of ZnO nanorods using low- energy femtosecond pulses
}

Cite as: J. Appl. Phys. 108, 043107 (2010); https://doi.org/10.1063/1.3468632

Submitted: 01 March 2010 . Accepted: 30 June 2010 . Published Online: 25 August 2010

Susanta Kumar Das, Mahua Biswas, Daragh Byrne, Martin Bock, Enda McGlynn, Markus Breusing, and Ruediger Grunwald

\section{ARTICLES YOU MAY BE INTERESTED IN}

Multiphoton absorption induced amplified spontaneous emission from biocatalystsynthesized ZnO nanorods

Applied Physics Letters 92, 233116 (2008); https://doi.org/10.1063/1.2927486

Multiphoton absorption pumped ultraviolet stimulated emission from ZnO microtubes Applied Physics Letters 91, 142109 (2007); https://doi.org/10.1063/1.2794776

Femtosecond pulse excited two-photon photoluminescence and second harmonic generation in $\mathrm{ZnO}$ nanowires

Applied Physics Letters 89, 042117 (2006); https://doi.org/10.1063/1.2236276

Applied Physics Reviews Now accepting original research 


\title{
Multiphoton-absorption induced ultraviolet luminescence of $\mathrm{ZnO}$ nanorods using low-energy femtosecond pulses
}

\author{
Susanta Kumar Das, ${ }^{1}$ Mahua Biswas, ${ }^{2}$ Daragh Byrne,${ }^{2}$ Martin Bock, ${ }^{1}$ Enda McGlynn, ${ }^{2}$ \\ Markus Breusing, ${ }^{1}$ and Ruediger Grunwald ${ }^{1, a)}$ \\ ${ }^{1}$ Max-Born-Institut für Nichtlineare Optik und Kurzzeitspektroskopie, Max-Born-Strasse 2a, D-12489 Berlin, \\ Germany \\ ${ }^{2}$ School of Physical Sciences, NCPST, Dublin City University, Glasnevin, Dublin 9, Ireland
}

(Received 1 March 2010; accepted 30 June 2010; published online 25 August 2010)

\begin{abstract}
Multiphoton-absorption (MPA) induced ultraviolet (UV) luminescence of $\mathrm{ZnO}$ nanorods grown by vapor phase transport was demonstrated using ultrafast excitation at pulse energies in the few nanojoules range, directly generated by a Ti:sapphire laser oscillator at wavelengths around $800 \mathrm{~nm}$. The dependence of the UV luminescence on the excitation density reveals a two-photon absorption process as the responsible excitation mechanism. The broad spectral bandwidth of the excitation pulses obviously promotes the feasibility of the observed two-photon channel. Theoretical estimates concerning the contribution of nonlinear absorbance strongly support the experimental findings. The essential conditions for proper utilization of this process are discussed. (C) 2010 American Institute of Physics. [doi:10.1063/1.3468632]
\end{abstract}

\section{INTRODUCTION}

Ultrafast multiphoton excitation of semiconductors by near infrared (NIR) photons has been proven to be an efficient way for the generation of highly energetic radiation compared to other frequency upconversion and shortwavelength laser schemes. In case of optically excited nanostructures, further specific properties can be exploited. Higher charge densities and electrostatic potential gradients caused by grain boundaries and surface inhomogeneities result in an increase in the total dipole moment and thus lead to significantly enhanced nonlinear optical effects. ${ }^{1}$ Therefore, particular attention is currently drawn to the investigation of structurally favored multiphoton excitation channels. With the availability of compact and highly intense femtosecond (fs) pump sources and in a view of various emerging applications utilizing such sources (as outlined below), systematic studies of specific excitation channels of wide-band gap semiconductors are of ever growing importance.

Because of its direct band gap and high exciton binding energy, $\mathrm{ZnO}$ is a promising candidate for novel optoelectronic and photovoltaic components. Among a remarkable variety of shapes of $\mathrm{ZnO}$ nanostructures (e.g., flakes, fibers, needles, and scales), ${ }^{2} \mathrm{ZnO}$ nanorods are of outstanding interest, motivated not only by their unique materials properties but also by the ease of preparing regular nanorod arrays of excellent optical quality (in terms of band edge emission intensity and bound exciton linewidths). The integration of $\mathrm{ZnO}$ nanorod structures with conventional NIR lasers is a promising approach to realize chip-level ultraviolet (UV) nanolasers. $^{3-6}$ Furthermore, by coupling NIR radiation through the "tissue optical window" (wavelength range 0.7-1.1 $\mu \mathrm{m}$ ) into nanowires or nanorods embedded in body tissue new methods of local photodynamic therapy can be enabled, if suitably high conversion efficiencies into the UV

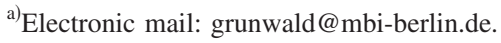

spectral range can be attained. ${ }^{7-10}$ Moreover, efficient multiphoton excitation channels open new prospects for a nonlinear processing of extremely short pulses (e.g., in twodimensional autocorrelation techniques). A detailed understanding of the luminescence properties and their dependence on fabrication-conditioned structural features is highly essential for all of these applications and is the main goal of the studies presented here.

Room temperature multiphoton-absorption (MPA) induced luminescence spectra of $\mathrm{ZnO}$ are generally found to display two characteristic bands, similar to those seen in single photon excited luminescence, specifically (i) a narrower near-band edge UV emission and (ii) a broader visible (VIS) band centered in blue-green spectral range. The UV band is composed of contributions from free-exciton recombination and its longitudinal-optical phonon replicas whereas the VIS band is caused by defect level emission (DLE) ${ }^{11}$ For the applications addressed above, the generation of DLE-free UV emission by multiphoton excitation processes is of major importance, in order to optimize the conversion efficiency to the UV range. As was pointed out in various reports by other groups, $\mathrm{ZnO}$ nanorods can meet this demand. ${ }^{7,12,13}$ Such works were performed with optical parametric oscillators as tunable excitation sources with center wavelengths between $520 \mathrm{~nm}$ and $700 \mathrm{~nm}$ (Refs. 12 and 13) or with amplified fs pulses from a Ti:sapphire laser emitting around $800 \mathrm{~nm}{ }^{7}$ Because of their compactness, lower cost and capability to work at high repetition rates $(\sim 75-100 \mathrm{MHz})$, Ti:sapphire oscillators could be an advantageous alternative if nanomaterials of sufficiently high efficiency were available.

In this report we demonstrate that it is feasible to obtain DLE-free UV luminescence from vapor phase transport (VPT)-grown $\mathrm{ZnO}$ nanorod arrays by fs pulse irradiation even at pulse energies in the few nanojoules range, directly emitted from a Ti:sapphire oscillator. Above band gap excitation is explained by two-photon absorption (2PA) and the 
possible influence of metallic nanoparticles remaining from the growth process are discussed. The excitation spectrum is well adapted to the tissue optical window for medical applications.

\section{EXPERIMENTAL}

The $\mathrm{ZnO}$ nanorods used in our experiments were grown by a VPT method using either (a) a vapor-liquid-solid (VLS) mechanism on a-plane (11-20) sapphire $\left(\mathrm{Al}_{2} \mathrm{O}_{3}\right)$ substrates with $\mathrm{Au}$ as a catalyst or (b) a vapor-solid (VS) mechanism on a two stage $\mathrm{ZnO}$ buffer layer grown on a quartz substrate using chemical bath deposition. ${ }^{14,15}$ For the type (a) samples, Au was evaporated with an equivalent thickness of $5 \mathrm{~nm}$. For type (b) samples growth took place directly upon the buffer layer with no Au catalyst employed; the details of the buffer layer growth are discussed in Ref. 15. The VPT growth for both sample types (a) and (b) was performed in a single-zone furnace within a horizontal quartz tube (outer diameter $4 \mathrm{~cm}$, inner diameter $3.7 \mathrm{~cm}$ ). High purity $\mathrm{ZnO}$ and graphite powders served as source materials. Equal parts $(0.06 \mathrm{~g}$ of each powder/1:1 ratio) of both powders were well mixed. Substrate and source powders were placed in a quartz boat at the central part of the furnace and processed at a temperature of $950{ }^{\circ} \mathrm{C}$. An Ar carrier gas with a flow rate of $90 \mathrm{SCCM}$ (SCCM denotes cubic centimeter per minute at STP) was present during the growth. The tube was open to the atmosphere at the exhaust end and thus growth occurred at atmospheric pressure. The residual atmospheric gas in the tube served as the source of oxygen. The duration of a complete growth cycle was $60 \mathrm{~min}$. The crystal structure of the nanorod array was analyzed using an X-ray diffractometer (Bruker AXS D8 advance texture diffractometer) and a scanning electron microscope (SEM) (JEOL, JSM-6400F). Type (a) samples showed a slightly reddish color under daylight illumination. This can be explained by the known effect that, during the growth process, the Au beads up into nanoscale droplets with a plasmon resonance in $\sim 600 \mathrm{~nm}$ range. Type (b) samples by contrast have a whitish appearance.

MPA-induced luminescence was mostly studied by exciting the nanorods with a Ti:sapphire laser system consisting of a diode-pumped solid-state laser Millennia (SpectraPhysics) and a Ti:sapphire laser oscillator (Femtosource) emitting linearly polarized 13 fs pulses at a repetition rate of $75.3 \mathrm{MHz}$ with a maximum average power of $313 \mathrm{~mW}$. The central wavelength was near $800 \mathrm{~nm}$ and the full-width-athalf-maximum (FWHM) bandwidth was $\sim 100 \mathrm{~nm}$. The base-to-base spectral range of the pulses is 710 to $890 \mathrm{~nm}$. The detailed spectral distribution of this pulse can be found in one of our earlier reports. ${ }^{16}$ The laser pulses were focused onto the samples with a lens of focal length, $f_{1}=20 \mathrm{~mm}$. The order of the nonlinear absorption process responsible for UV luminescence excitation was determined by varying the pulse intensity with a set of neutral density filters. The pulse durations at different stages of propagation were retrieved from spectral phase data measured with an LX-SPIDER system (spectral phase interferometry for direct electric-field reconstruction with an extended nonlinear crystal). The values after passing through the filters (i) and behind the lens (ii) were

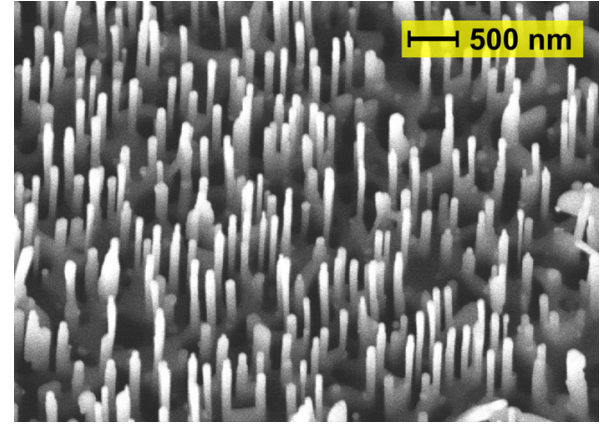

FIG. 1. (Color online) SEM images of the $\mathrm{ZnO}$ nanorod arrays grown by VLS mechanism.

found to be 23.5 fs and 29 fs, respectively. For determining the intensity dependence, the pulse energy was varied between $25 \mathrm{pJ}$ and $3.09 \mathrm{~nJ}$. The corresponding peak power densities were estimated to be in the range between 0.28 and $33.95 \mathrm{GW} / \mathrm{cm}^{2}$ and we note that the aforementioned pulse broadening effects by lenses and neutral density filters were taken into account in these calculations. The angle of incident of the focus cone was either $0^{\circ}$ or $60^{\circ}$ to the surface normal. The later angle was used for the polarization dependence study of the generated signals. The generated luminescence was collected in transmission direction by a lens of focal length, $f_{2}=18 \mathrm{~mm}$ or $50 \mathrm{~mm}$. (The later was used in polarization dependence study). The luminescence spectrum was separated from residual pump radiation by a BG39 color glass filter and analyzed with a fiber-coupled grating spectrometer (HR 2000, Ocean Optics) and a high sensitivity electron multiplier charge coupled device (EMCCD) based spectrometer (Newton, Andor Technology). The EMCCD based spectrometer was mainly used for the incident power density dependence of the generated signal. All experiments were carried out at room temperature (the EMCCD was internally cooled down to $-75^{\circ} \mathrm{C}$ electronically).

\section{RESULTS AND DISCUSSION}

Figure 1 shows an SEM image of the type-(a), VLSgrown, nanorods which have an average diameter of $\sim 90 \mathrm{~nm}$. The nanorods are preferentially well aligned in a direction perpendicular to the substrate. The $\mathrm{x}$-ray diffraction (XRD) analysis data of the nanorods are presented in Fig. 2.

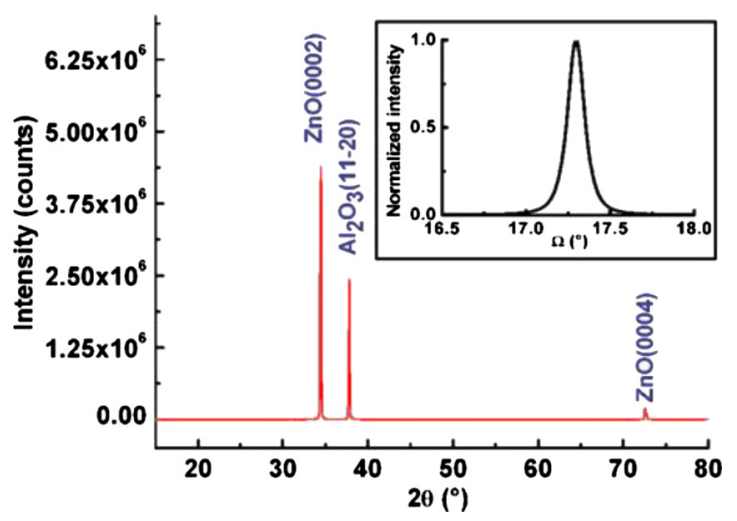

FIG. 2. (Color online) XRD; (inset: rocking curve) for the $\mathrm{ZnO}$ nanorod arrays grown by VLS mechanism. 


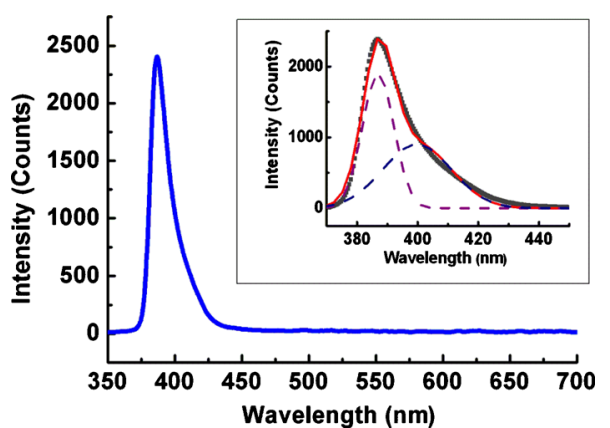

FIG. 3. (Color online) The emission spectra in $\mathrm{ZnO}$ nanorods grown by VLS mechanism when excited by a broadband (FWHM=103 nm) fs pulse having central wavelength at $\sim 800 \mathrm{~nm}$. The pulse energy was $4.16 \mathrm{~nJ}$ as directly extracted from Ti:sapphire laser oscillator without amplifier (peak power $36.75 \mathrm{GW} / \mathrm{cm}^{2}$ ) [Inset: the multiple-Gaussian fits (dotted curves) show the UV luminescence (peak $=386 \mathrm{~nm}$ ) and SHG (peak=403 nm) components of this spectrum. The solid line represent the sum of both fit and the scattered rectangular points display the real spectrum.]

The two peaks at $\sim 34.5^{\circ}$ and $72.5^{\circ}$ represent the (0002) and (0004) reflections of crystalline $\mathrm{ZnO}$ and indicate clearly a predominant orientation of the nanorods along the c-axis direction. Another peak at $\sim 37.7^{\circ}$ appears due to the (11-20) plane of the sapphire substrate. The rocking curve of the $\mathrm{ZnO}$ (0002) reflection from this sample can be found in the inset of Fig. 2. The FWHM of the rocking curve is 0.12 degrees. This very small value indicates the excellent alignment of the nanostructures deposited with c-axis orientation perpendicular to the sapphire substrate surface. The type (b) samples have similar appearance in SEM and have a slightly broader rocking curve in XRD.

The typical emission spectrum from the type (a) $\mathrm{ZnO}$ nanorods at room temperature for a pulse energy of $4.16 \mathrm{~nJ}$ of the exciting laser (peak power $=36.75 \mathrm{GW} / \mathrm{cm}^{2}$, no use of filter set) is shown in Fig. 3. The spectrum was found to be concentrated in the UV-region and defect-related VIS emission is completely absent in this case. The UV spectrum appears to be composed of two contributions related to (a) band edge UV luminescence and (b) second harmonic generation (SHG). The individual contributions can be deconvolved using a double-Gaussian fit (with a dc baseline) of the spectrum. From the fit curves, luminescence emission and peak of the SHG were estimated to be located at wavelengths of $\sim 386 \mathrm{~nm}$ and $400 \mathrm{~nm}$, respectively, (inset of Fig. 3). Similar MPA-induced luminescence spectra were also observed when the sample was excited with more powerful (few microjoules) amplified pulses with pulse durations about 150 fs. In that case, however, the luminescence peak was found to be well distinguishable from the SHG peak due to the narrower spectral bandwidth $(\sim 10 \mathrm{~nm})$ of the input pulses. It is worth to be pointed out that under the excitation of the fs pulses, the coexistence of MPA-induced luminescence and SHG is a common phenomenon in $\mathrm{ZnO}$. For example, Dai et al. ${ }^{17}$ presented a detailed report on this topic. Unlike in our case, however, their study was performed with $\mathrm{ZnO}$ single crystals where the luminescence mostly appears because of defect levels. A coexistence of SHG and MPAinduced luminescence in $\mathrm{ZnO}$ nanorods was demonstrated by

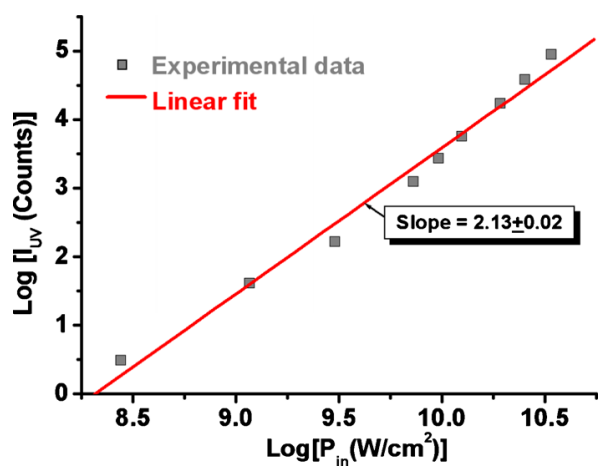

FIG. 4. (Color online) Double-logarithmic plot of the UV luminescence signal as a function of the laser peak power. Scattered points: experimental data; solid line: linear fit.

Zhang et $_{\text {al. }}{ }^{7}$ at significantly different conditions (higher excitation level by applying amplified fs pulses, much higher pulse duration).

The dependence of the logarithmic UV luminescence signal $I_{\mathrm{UV}}$ (i.e., the integrated area underneath the peak at $386 \mathrm{~nm}$ ) on the logarithmic incident peak power $P_{\text {input }}^{\text {Peak Power }}$ is displayed in Fig. 4. A linear fit curve representing the equation

$$
\log \left(I_{\mathrm{UV}}\right)=A+n \log \left(P_{\text {input }}^{\text {Peak Power }}\right)
$$

to this variation delivers the nonlinear coefficient $n=2.13 \pm 0.02$ which is related to the order of the excitation process. (Please note: a similar analysis was also carried out for the SHG peak for the confirmation of the second order process.) Here, the value of $n$ indicates a predominantly 2PA-related channel. This agrees well with the expected excitation channel, given the spectral width of the source pulses. From the literature it is known that $\mathrm{ZnO}$ can show strong room temperature 2PA even at a wavelength of 770 $\mathrm{nm} .{ }^{18}$ Thus, the short-wavelength region of the incident spectrum below $770 \mathrm{~nm}(\sim 25 \%$ of the power of the input pulse) is responsible for the 2PA-induced luminescence in our case. Spectral contributions at longer wavelengths $(\sim 75 \%$ of the power) will contribute to 3PA-induced luminescence which is several orders of magnitude weaker than 2PA-induced one $^{18}$ and thus the 2PA process dominates. It should be noted that the double-Gaussian fitting procedure performed for each laser peak power value proved to be a reliable method of curve analysis with consistent values of fitted peak position for both contributions found across the entire range of input powers. The fit curves for the highest $\left(33.95 \mathrm{GW} / \mathrm{cm}^{2}\right)$, intermediate $\left(9.62 \mathrm{GW} / \mathrm{cm}^{2}\right)$, and lowest $\left(0.28 \mathrm{GW} / \mathrm{cm}^{2}\right)$ value of peak power density used for the estimation $n$ are shown in Figs. 5(a)-5(c), respectively. Similar robust fits (details shown in Table I) for both UV luminescence and SHG were found for all power values thus giving ample confidence in the values extracted. Figure 5 also clearly shows the evolution of the UV luminescence peak (peak at $\sim 385 \mathrm{~nm}$ ) with the increasing peak power density. At low peak power density, the UV luminescence is very weak compared to the SHG signal and with increasing intensity it dominates. 


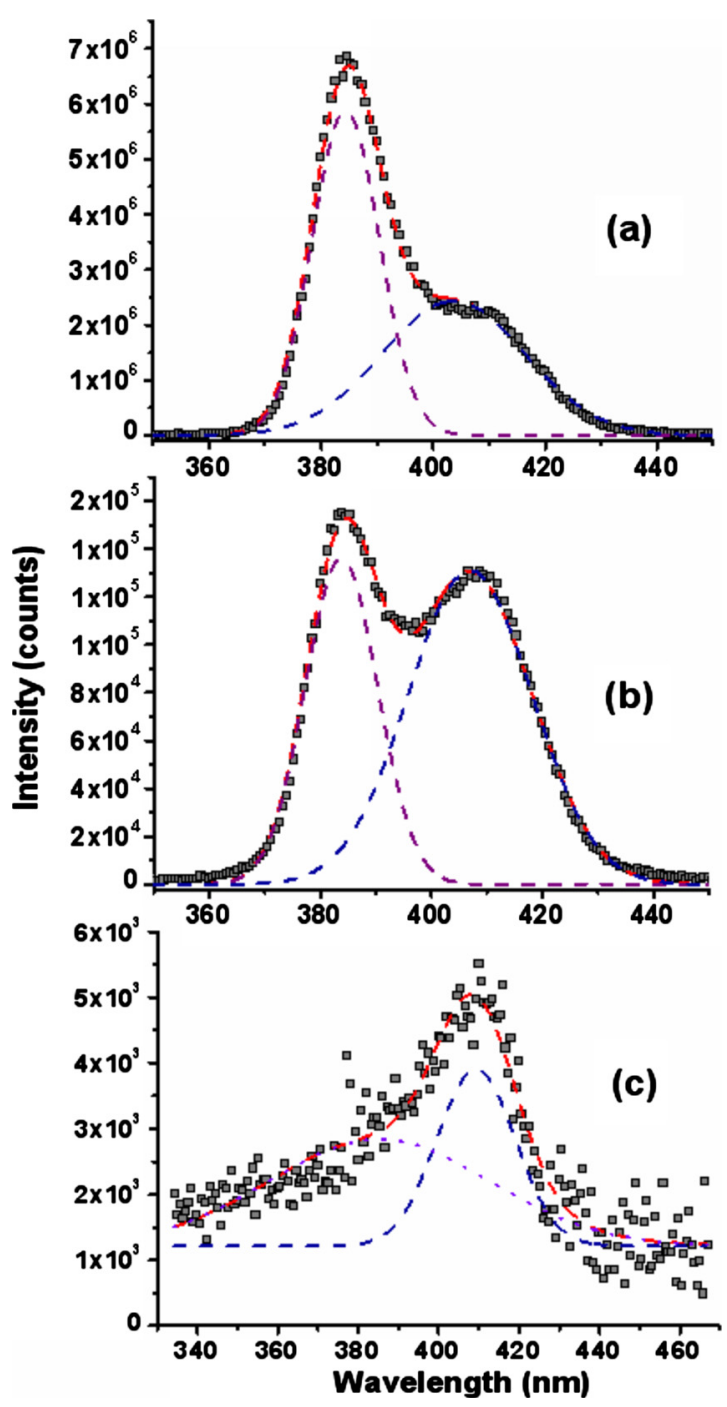

FIG. 5. (Color online) MPA-induced emission spectra generated in $\mathrm{ZnO}$ nanorods grown by VLS mechanism at different excitation densities. The scattered rectangular points represent the original signal. Dashed curves: fit curves for UV and SHG contributions obtained by multi-Gaussian decomposition algorithm. The upper dashed curves represent the sum of both fit. The excitation densities were $33.95,9.62$, and $0.28 \mathrm{GW} / \mathrm{cm}^{2}$ for (a), (b), and (c), respectively.

The dominance of the 2PA over the 3PA for broadband ultrashort pulses with central wavelengths near $800 \mathrm{~nm}$ in $\mathrm{ZnO}$ can also be verified through the theoretical estimation of nonlinear absorbances. For the typical conditions in our experiments (13 fs pulse duration, $800 \mathrm{~nm}$ center wavelength, $\mathrm{ZnO}$ material), we defined the normalized twophoton absorbance $\left(A_{2}\right)$ and three-photon absorbance $\left(A_{3}\right)$ (compare to Ref. 18) as

$$
\begin{aligned}
& A_{2}=\sum_{\lambda_{i}=710 \mathrm{~nm}}^{770 \mathrm{~nm}} \frac{\alpha_{2}\left(\lambda_{i}\right) \omega\left(\lambda_{i}\right) L_{\mathrm{eff}}\left(\lambda_{i}\right) I_{0}}{2^{3 / 2}}, \\
& A_{3}=\sum_{\lambda_{i}=770 \mathrm{~nm}}^{890 \mathrm{~nm}} \frac{\alpha_{3}\left(\lambda_{i}\right) \omega\left(\lambda_{i}\right)^{2} L_{\mathrm{eff}}^{\prime}\left(\lambda_{i}\right) I_{0}^{2}}{3^{3 / 2}} .
\end{aligned}
$$

The operands in the above expressions represent the of nonlinear absorption terms of Z-scan theory (open aperture) for the radiation of wavelength $\lambda_{i}$ and the summations over them
TABLE I. FWHM and peak position of UV-PL and SHG at different peak power levels.

\begin{tabular}{ccccccc}
\hline \hline & \multicolumn{3}{c}{ UV } & & \multicolumn{2}{c}{ SHG } \\
\cline { 2 - 3 } \cline { 5 - 6 } $\begin{array}{c}\text { Peak power } \\
\left(\mathrm{GW} / \mathrm{cm}^{2}\right)\end{array}$ & $\begin{array}{c}\text { Peak position } \\
(\mathrm{nm})\end{array}$ & $\begin{array}{c}\text { FWHM } \\
(\mathrm{nm})\end{array}$ & & $\begin{array}{c}\text { Peak position } \\
(\mathrm{nm})\end{array}$ & $\begin{array}{c}\text { FWHM } \\
(\mathrm{nm})\end{array}$ \\
\hline 33.95 & 384.37 & 11.76 & & 403.5 & 25 \\
25.36 & 384.02 & 12.06 & & 405 & 22.9 \\
19.24 & 383.63 & 11.65 & & 404 & 24.8 \\
12.53 & 383.8 & 12.3 & & 407 & 21.6 \\
9.62 & 383.8 & 12.8 & & 407 & 22.2 \\
7.29 & 384.12 & 13.2 & & 407.5 & 22.7 \\
3.02 & 386.0 & 17.97 & & 409.4 & 19.0 \\
1.16 & 387.75 & 57.46 & & 407.9 & 21.5 \\
0.28 & 385.27 & 54.97 & & 409.31 & 19.32 \\
\hline \hline
\end{tabular}

(in proper spectral interval) are taken to represent the strength of nonlinear absorptions for the whole broad spectra of our $13 \mathrm{fs}$ pulses. Here $I_{0}$ describes the peak power density incident on the sample, $\omega\left(\lambda_{i}\right)$ is the statistical weight of the $\lambda_{i}$ th component of the broadband spectrum of the ultrashort pulse, and $\alpha_{2}\left(\lambda_{i}\right)$ and $\alpha_{3}\left(\lambda_{i}\right)$ are the 2PA- and 3PAabsorption coefficients at the wavelengths $\lambda_{i}$, respectively. The parameter $L_{\text {eff }}\left(\lambda_{i}\right)$ and $L_{\text {eff }}^{\prime}\left(\lambda_{i}\right)$ stands for the effective thickness of the sample for 2PA and 3PA processes, respectively,

$$
\begin{aligned}
& L_{\mathrm{eff}}\left(\lambda_{i}\right)=\frac{1-\exp \left[-\alpha_{0}\left(\lambda_{i}\right) L\right]}{\alpha_{0}}, \\
& L_{\mathrm{eff}}^{\prime}\left(\lambda_{i}\right)=\frac{1-\exp \left[-2 \alpha_{0}\left(\lambda_{i}\right) L\right]}{2 \alpha_{0}},
\end{aligned}
$$

with the linear absorption coefficient $\alpha_{0}\left(\lambda_{i}\right)$ of the material at the wavelength $\lambda_{i}$ and the thickness of the sample $L$. Figure 6 shows the variation in the two- and three-photon absorbances with the peak power density. The dotted lines specify the working range of peak power density in our case. Here the values of $\omega\left(\lambda_{i}\right)$ were taken in the spectral interval of $\sim 0.45 \mathrm{~nm}$. The corresponding values of $\alpha_{2}\left(\lambda_{i}\right)$ and $\alpha_{3}\left(\lambda_{i}\right)$ were extracted from data reported for $\mathrm{ZnO}$ in Ref. 18 with the nonlinear fit curves. The effective thickness of the sample is taken to the real thickness of sample because of the negligible linear absorption of the input radiation. This thickness is taken to be $500 \mathrm{~nm}$ ( length of the nanorods) for the

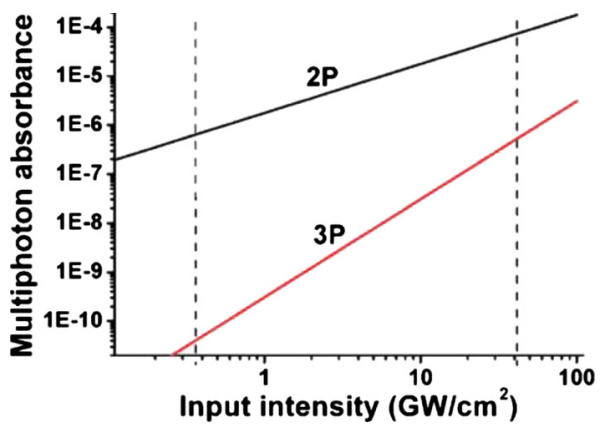

FIG. 6. (Color online) Variation in the two-photon and three-photon absorbance for excitation with a 13 fs pulse with a central wavelength of $800 \mathrm{~nm}$. 


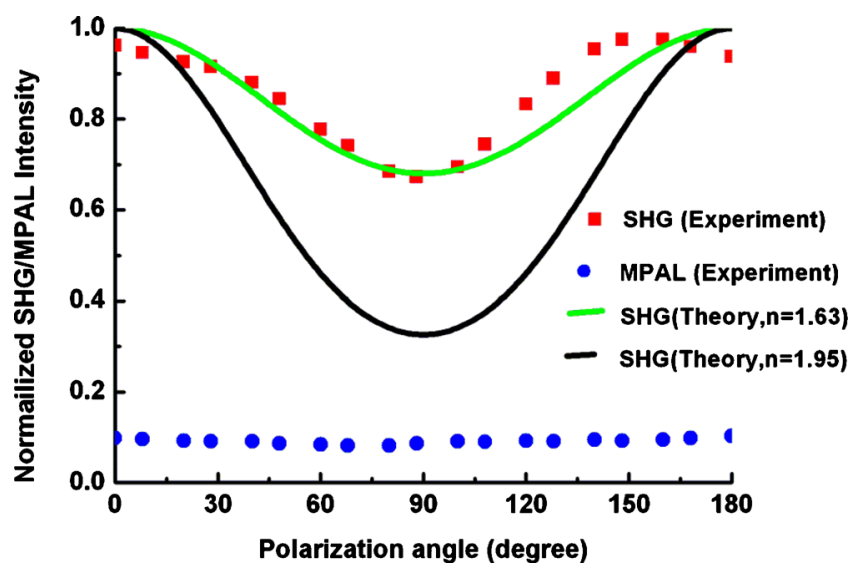

FIG. 7. (Color online) Polarization dependence behavior of SHG and MPAinduced luminescence signal (MPAL).

numerical simulation. The curves in Fig. 6 clearly indicate that within the relevant working range (marked by dotted lines) the two-photon absorbance significantly exceeds the three-photon absorbance, by several orders of magnitude. This lends further support to the conclusion that the MPAinduced luminescence observed in our experiments is mainly due to a $2 \mathrm{PA}$-process.

In order to further investigate the behavior of MPAinduced luminescence and SHG we studied polarization dependence by following the method suggested by Yang et al. ${ }^{19}$ The experimental result is shown in Fig. 7 (rectangular red dots) for a range of a $180^{\circ}$ rotation of the polarization of the input beam. First, the SHG signal decreases gradually until the polarization was rotated by $90^{\circ}$. With further rotation, the SHG gradually increases until $180^{\circ}$. The quantitative variation in the SHG agrees well with the behavior expected from theoretical simulation. $\mathrm{ZnO}$ exhibits a $6 \mathrm{~mm}$ point group symmetry. ${ }^{20}$ For excitation wavelengths far from the resonance, the SHG is mainly controlled by two independent second order susceptibility components $\chi_{x z x}^{(2)}=\chi_{y z y}^{(2)}=\chi_{x x z}^{(2)}$ $=\chi_{y y z}^{(2)}=\chi_{z x x}^{(2)}=\chi_{z y y}^{(2)}$ and $\chi_{z z z}^{(2)}$. The variation in the p-polarized second harmonics with respect to the polarization angle can be estimated by the expression ${ }^{21}$

$$
\begin{aligned}
P\left(\theta^{\prime}, \alpha\right)= & A\left\{\operatorname { c o s } ^ { 2 } \alpha \left[3 \chi_{z x x}^{(2)} \cos ^{2}\left(\theta^{\prime}-\theta_{0}^{\prime}\right)+\chi_{z z z}^{(2)} \sin ^{2}\left(\theta^{\prime}\right.\right.\right. \\
& \left.\left.\left.-\theta_{0}^{\prime}\right)\right]+\chi_{z x x}^{(2)} \sin ^{2} \alpha\right\}^{2},
\end{aligned}
$$

where $\alpha=$ polarization angle, $\sin \theta^{\prime}=\sin \theta / n \theta=$ angle of incidence, $\mathrm{n}=$ refractive index of the material and $\theta_{0}^{\prime}=$ angle of inclination of the nanorods relative to (in our case) the c-axis. The normalized SHG power calculated as a function of the polarization angle is shown in the same Fig. 6 (solid lines). The angles $\theta$ and $\theta_{0}^{\prime}$ for this simulation were chosen to be $60^{\circ}$ and $0^{\circ}$, respectively, corresponding to the real experimental conditions. The values for $\chi_{z z z}^{(2)}$ and $\chi_{z x x}^{(2)}$ were set to $-14.0 \mathrm{pm} / \mathrm{V}$ and $4.2 \mathrm{pm} / \mathrm{V}$ (supposing that data for bulk $\mathrm{ZnO}$ crystal $^{20}$ can be applied). The experimental results were found to fit the theoretical variation relatively well if a refractive index of $n=1.63$ is assumed (green solid line) whereas the approximation fails for $n=1.95$ (bulk $\mathrm{ZnO}$ ). Obviously, the relatively small fill factor of the nanorods results in an effective index lowered by about $16 \%$. On the contrary, the MPA-induced luminescence (blue dots) does not show any definite behavior. This had largely to be expected because of the similarity of $\mathrm{ZnO}$ absorption at room temperature for both $\mathrm{E} \| \mathrm{c}$ and $\mathrm{E} \perp \mathrm{c}$ polarizations ${ }^{22}$ and thermalization processes.

A high degree of perfection in the material quality (i.e., purity, crystallinity, etc.) is one of the most important preconditions for the realization of 2PA-induced UV luminescence. This was verified through a comparative MPAinduced luminescence study of the VLS-grown $\mathrm{ZnO}$ nanorods with other materials like a commercial $\mathrm{ZnO}$ single crystal (c-axis grown, $500 \mu \mathrm{m}$ thickness, CrysTech $\mathrm{GmbH}$ ) and $\mathrm{ZnO}$ nanorods of similar dimensions grown by low temperature chemical bath method. ${ }^{23}$ The $\mathrm{ZnO}$ bulk crystal was found to show only very weak UV emission and dominant DLE, whereas the chemical bath grown $\mathrm{ZnO}$ nanorods (whose optical quality was verified to be inferior to the VLSgrown nanorods in a photoluminescence study) yield no detectable luminescence under the same experimental conditions.

In order to check if surface plasmonic effects due to Au nanodroplets present at the tips or bases of the type-(a) VLSgrown material could have played a role in generating DLE free UV luminescence ${ }^{24}$ we investigated a different set of VS-grown samples [type (b)]. All of these samples were found to show excellent DLE-free UV luminescence and the strength of the luminescence and variation in emission intensity with the excitation power were fully comparable to the VLS-grown samples. We also note here that a further set of VPT-grown type (b) samples were coated with a thin Au layer (equivalent thickness $5 \mathrm{~nm}$ ), post nanorod growth, in order to enable direct comparisons of the effects of $\mathrm{Au}$ nanoparticles/films. In all cases we observed a reduction in UV luminescence consistent with a simple masking effect. This indicates that surface plasmonic effects caused by the $\mathrm{Au}$ nanodroplet catalysis have hardly played any role in our case, in contrast to effects seen in samples with significant levels of DLE. ${ }^{24}$

Finally we emphasize that the 2PA-induced UV luminescence observed from $\mathrm{ZnO}$ nanorods was excited with high repetition rate $(75.3 \mathrm{MHz})$ laser pulses from the oscillator. Due to this fact, the time integrated signal of the UV luminescence was found to be high enough in our case for reliable detection even with uncooled detector arrays working at integration time constants of a few milliseconds. This implies that our approach, utilizing high repetition rate unamplified fs pulses as excitation sources, has the potential for the generation of high average power UV luminescence in $\mathrm{ZnO}$ nanorods. Obviously, the combination of highly efficient broadband excitation with extremely short pulses and high repetition rates enables sufficiently high 2PA signals for use in practical applications, even from compact, low-cost, fs laser sources.

Moreover, it is expected that with sources operating at slightly downshifted center wavelengths ${ }^{25} \mathrm{UV}$ luminescence could be generated via 2PA in VPT-grown $\mathrm{ZnO}$ nanorods with considerably higher efficiency. The use of ultrabroadband Ti:sapphire laser pulses could also be another option for the generation of efficient 2PA induced UV luminescence. 
For example, in our preliminary investigation ${ }^{26}$ we found that more than $55 \%$ of the energy of a 7 fs pulse, with central frequency at $\sim 800 \mathrm{~nm}$ and a FWHM of $\sim 150 \mathrm{~nm}$, from a suitable oscillator can facilitate $2 \mathrm{PA}$.

\section{CONCLUSIONS}

To conclude, we have demonstrated the generation of UV luminescence in $\mathrm{ZnO}$ nanorods grown by VPT with ultrashort fs pulses at $800 \mathrm{~nm}$ directly from a Ti:sapphire laser oscillator (few nanojoules range). The experimental findings strongly indicate a 2PA-induced excitation as the mechanism responsible, which is enabled by the broad spectral range of the input pulses. This assumption was verified by theoretical investigations. Furthermore it was shown that surface plasmon effects play no significant role for the DLE-free UV luminescence in our samples. Due to the highly repetitive operation of the laser oscillator, the low-pulse-energy approach used in our experiments has good potential for the generation of high average power UV luminescence in $\mathrm{ZnO}$ nanorods. The 2PA-induced, spatially localized, UV luminescence in $\mathrm{ZnO}$ nanorods excited with NIR wavelengths in the tissue optical window promises to be a useful tool for photodynamic therapy and related applications. Systematic studies of 2PA-induced UV luminescence with spectral management and larger bandwidths will be the subject of future investigations.

\section{ACKNOWLEDGMENTS}

The authors thank Professor T. Elsaesser, M. Tischer, and C. Poppe (Max-Born-Institute), Professor F. Güell (University Barcelona, Spain), Professor F. Henneberger and Mr. S. Kalusniak (Institut für Physik, Humboldt-Universität zu Berlin), and Professor F. Rotermund (Ajou University, Korea) for useful discussions and assistance. The work was financially supported in part by DFG (Contract No. 1782/121). D. Byrne and E. McGlynn acknowledge the Science Foundation Ireland (SFI) for a Strategic Research Cluster grant (Functional Oxides and Related Materials for Electronics, FORME). M. Biswas and E. McGlynn are grateful for support in frame of SFI Research Frontiers Programme
(Grant No. 06/RFP/PHY052).

${ }^{1}$ J. Ebothe, I. V. Kityk, and I. Fuks-Janczarek, Appl. Surf. Sci. 252, 5763 (2006).

${ }^{2}$ A. B. Djurišić and Y. H. Leung, Small 2, 944 (2006).

${ }^{3}$ Z.-W. Dong, C. F. Zhang, K.-J. Liu, Y. L. Yan, H. Deng, and S. H. X. Qian, Front. Phys. China 3, 181 (2008).

${ }^{4}$ M. H. Huang, S. Mao, H. Feick, H. Q. Yan, Y. Y. Wu, H. Kind, E. Weber, R. Russo, and P. D. Yang, Science 292, 1897 (2001).

${ }^{5}$ C. F. Zhang, Z. W. Dong, G. J. You, S. X. Qian, and H. Deng, Opt. Lett. 31, 3345 (2006)

${ }^{6}$ C. F. Zhang, Z. W. Dong, K. J. Liu, Y. L. Yan, and S. X. Qian, Appl. Phys. Lett. 91, 142109 (2007).

${ }^{7}$ C. Zhang, F. Zhang, S. Qian, N. Kumar, J. Hahm, and J. Xu, Appl. Phys. Lett. 92, 233116 (2008).

${ }^{8}$ D. Sridhar, X. Jining, J. K. Abraham, and V. K. Varadan, Proc. SPIE 6528 , 65281L (2007).

${ }^{9}$ C. Baratto, E. Comini, G. Faglia, G. Sberveglieri, M. Zha, and A. Zappettini, Sens. Actuators B 109, 2 (2005).

${ }^{10}$ C. Sridhar, J. N. Xie, J. K. Abraham, and V. K. Varadam, Proc. SPIE 6172, 617208 (2006).

${ }^{11}$ D. C. Dai, S. J. Xu, S. L. Shi, and M. H. Xie, Opt. Lett. 30, 3377 (2005).

${ }^{12}$ G. Zhu, C. Xu, J. Zhu, C. Lu, Y. Cui, and X. Sun, J. Nanosci. Nanotechnol. 8, 5854 (2008).

${ }^{13}$ C. Zhang, F. Zhang, and J. Xu, Conference on Lasers and Electro-Optics/ Quantum Electronics and Laser Science, 2008.

${ }^{14}$ L. E. Greene, M. Law, D. H. Tan, M. Montano, J. Goldberger, G. Somorjai, and P. Yang, Nano Lett. 5, 1231 (2005).

${ }^{15}$ D. Byrne, E. McGlynn, M. O. Henry, K. Kumar, and G. J. Hughes, Thin Solid Films 518, 4489 (2010).

${ }^{16}$ S. K. Das, M. Bock, C. O’Neil, R. Grunwald, K. Lee, H. Lee, S. Lee, and F. Rotermund, Appl. Phys. Lett. 93, 181112 (2008).

${ }^{17}$ D. C. Dai, S. J. Xu, S. L. Shi, M. H. Xie, and C. M. Che, IEEE Photonics Technol. Lett. 18, 1533 (2006).

${ }^{18}$ J. He, Y. Qu, H. Li, J. Mi, and W. Ji, Opt. Express 13, 9235 (2005).

${ }^{19}$ H. Yang, S. J. Xu, Q. Li, and J. Zhang, Appl. Phys. Lett. 88, 161113 (2006).

${ }^{20}$ Y. R. Shen, The Principles of Nonlinear Optics (Wiley, New York, 1984), p. 101.

${ }^{21}$ W. P. Lin, P. M. Lundquist, G. K. Wong, E. D. Rippert, and J. B. Ketterson, Appl. Phys. Lett. 63, 2875 (1993).

${ }^{22}$ W. Y. Liang and A. D. Yoffe, Phys. Rev. Lett. 20, 59 (1968).

${ }^{23}$ T. Mahalingam, K. M. Lee, K. H. Park, S. Lee, Y. Ahn, J.-Y. Park, and K. H. Koh, Nanotechnology 18, 035606 (2007).

${ }^{24}$ H. Y. Lin, C. L. Cheng, Y. Y. Chou, L. L. Huang, Y. F. Chen, and K. T. Tsen, Opt. Express 14, 2372 (2006).

${ }^{25}$ J. M. Shieh, T. C. Huang, K. F. Huang, C. L. Wang, and C. L. Pan, Opt. Commun. 156, 53 (1998).

${ }^{26}$ See supplementary material at http://dx.doi.org/10.1063/1.3468632 for the typical input and output spectrum for $7 \mathrm{fs}$ laser pulses. 